\title{
Energy Efficient Wireless Sink Node for Monitoring of Snow Environment
}

\author{
Rajesh Kumar Garg ${ }^{1,2}$, Surender Kumar Soni ${ }^{3}$ \\ ${ }^{1}$ Department of E \& CE, National Institute of Technology, Hamirpur/ India \\ ${ }^{2}$ DGRE, Himparisar, Plot No.-1, Sector-37A, Chandigarh/ India \\ ${ }^{3}$ Department of E \& CE, National Institute of Technology, Hamirpur/ India
}

\begin{abstract}
Article History: Received: 10 November 2020; Revised: 12 January 2021; Accepted: 27 January 2021; Published online: 05 April 2021

Abstract: Conventionally, sink node is considered to have large hardware and energy resources; however, many times sink node is working in same conditions as source nodes, especially when deployed for monitoring of the snow environment. In this paper, an effort has been made to practically realize a sink node which is energy efficient and cost effective for monitoring applications. To save energy, the Main Power Module is designed to provide controlled powers to sensors and sub-modules. The paper discusses design aspects of the sink node and its long-term field evaluation with environmental sensors, especially the Snow Depth Sensor of MaxBotix. Field performance of Snow Depth Sensor has been enhanced by Euclidean Minimum Distance filter which improved the correlation of data to 0.997 . The proposed design helps to achieve energy consumption of $42.72 \mathrm{mWh}$ which is significantly lower than the previous work. The reliable working of the sink node in the long-term field evaluation indicates that snow environment can be monitored at less expense of energy by employing proposed sensors and the specially designed sink node.
\end{abstract}

Keywords: GSM, Monitoring, Sink node, Snow depth, WSN

\section{Introduction}

Wireless Sensor Networks (WSNs) have been conceptualized as the collection of large number of inexpensive nodes which have huge potential to monitor the environmental parameters at low cost. According to Akyildiz (2002), the wireless sensor nodes have limited resources in terms of memory, processing power, communication range and energy. The communication module onboard WSN node has small range, so by utilizing various energy efficient routing protocols (Heinzelman, Chandrakasan, \& Balakrishnan, 2000; Manjeshwar, \& Agrawal, 2001; Heinzelman, Chandrakasan, \& Balakrishnan, 2002; Manjeshwar, \& Agrawal, 2002; Chen et al., 2008; Kandris at al., 2009; Pantazis, Nikolidakis, \& Vergados, 2013; Yang, \& Cardei, 2010), the raw/ aggregated data are sent to the neighboring node, sink node or directly to the central station (Al-Karaki, Ul-Mustafa, \& Kamal, 2004). They are conceptually deployable in mass scale, spatially and temporally for monitoring the diverse range of applications including environment, waterways, forests, sensitive installations, buildings, security and battlefield scenarios (Corke et al., 2010; Oliveira, \& Rodrigues, 2011; Sardini, \& Serpelloni, 2011). For snow environment applications, Erlacher (2016) has embedded wireless sensor nodes in the snowpack before release of avalanches for better understanding of inner movement of snow-mass. The comparative evaluation of state-of-the-art ultrasonic snow depth sensors has been carried out by installing the sensors of M/s Campbell Scientific (SR-50) and M/s Judd Communications at nine sites across the United States (Ryan, Doesken, \& Fassnacht, 2008). These sensors are field proven, but they are expensive and consume more energy. Energy consumption has been an important concern in field based WSN applications.

The researchers (Alippi et al., 2009; Raghunathan, Ganeriwal, \& Srivastava, 2006) have focused on maximizing the lifetime of nodes, which derive energy primarily from the small capacity batteries. Simjee and Chou (2008) used super-capacitors and efficient rechargeable batteries in sensor nodes as they can be replenished from natural resources in the deployed areas by tapping solar power, wind energy and vibration energy (Jiang, Polastre, \& Culler, 2005; Sharma, Mukherji, \& Joseph, 2010). Efficient charging of storage devices has been reported by tracking Maximum Power Point between the source and charger (Subudhi, \& Pradhan, 2013; Park, \& Chou, 2006). Apart from improvements in energy storage devices and tapping mechanisms, the researchers (Sinha, \& Chandrakasan, 2001; Kompis, \& Sureka, 2010) have also devised power management techniques to lower the energy requirements of the network without compromising the end purpose of deployment. Mirza, Owrang, \& Schurgers (2005) have reported sample-and-send systems with many nodes and operational durations from days to years, whilst other researchers (Bai, \& Tepedelenlioglu, 2008; Khalil et al., 2017; Kolios et al., 2016) envisaged to send data only on occurrence of events. So, energy consumption has to be minimized in the deployment of Wireless Sensor Nodes. The source nodes of WSNs are energy efficient but they have lower hardware resources. The recorded data of source nodes are processed and disseminated to central station through sink node; thereby requiring more resources. Standard Arduino Mega Board meets this requirement of practical sink node, but consumes $\approx 336 \mathrm{mWh}$ of energy in power down mode. Usually, it is presumed that source nodes of WSNs have energy constraint but sink node has large resources in terms of energy, computational power and hardware features. However, energy consumption remains a challenge when deployed for environmental 
monitoring of snow bound regions of Indian Himalayas where all nodes are subjected to same environmental conditions.

In the past, conventional nodes have been designed with other popular microcontrollers for various types of monitoring applications. Gutierrez et al. (2014) developed automated irrigation system by monitoring the soil moisture and temperature of root zone of plants. The system was built around PIC24FJ64GB004 microcontroller, which had deployed Soil Moisture (VH400), Temperature Sensor (DS1822) and water pumps. The system was tested in a crop field for 136 days in the temperature range of $15^{\circ} \mathrm{C}$ to $38^{\circ} \mathrm{C}$. The microcontroller-based monitoring system consumed $80 \mathrm{mAh}(360 \mathrm{mWh})$ in operational mode excluding the power of water pumps. Yan, Sun, \& Qian (2013) presented energy aware sensor node design using MSP430F microcontroller. This paper presented "node-level energy saving" by adaptive transmission, power setting, periodic sleep/wake-up scheme, and "network-level energy saving" by adaptive network configuration. The power saving has been confirmed by experimental tests conducted in ambient conditions of greenhouse environment. Bengherbia et al. (2016) proposed FPGA based WSN sink node. The proposed architecture for the Sink node was built on MicroBlaze soft-core processor, and XBee communication module for communication. The proposed node was tested and implemented on the Digilent Basys3 Artix-7 FPGA board. Test application for temperature measurement $\left(\approx 20^{\circ} \mathrm{C}\right)$ in ambient conditions had been developed to ensure the proper functioning of the sink node. The proposed FPGA board was operating with power supply of $5000 \mathrm{mWh}$, which is very large for the standalone applications of snow monitoring.

Fisher, \& Kebede (2010) had proposed microcontroller-based system for crop monitoring. They developed an automated system based on PIC16F88 with solid-state components for handling clock/calendar, sensor power, and data storage and retrieval functions. Analog Soil Moisture Sensor (Watermark 200-SS), Digital Temperature Sensor (TC74), Analog Temperature Sensor (LM35) and Digital Infrared Thermometer (MLX90614) had been integrated with the system for crop monitoring. The system was used to monitor crop of corn and air temperature between $18^{\circ} \mathrm{C}$ to $37^{\circ} \mathrm{C}$ was recorded in the evaluation period. The proposed system did not have communication module and had limited ports which are a major requirement for the sink node. Yang, \& Tian (2013) proposed the design of sink node for environmental monitoring which consumed $368.32 \mathrm{mAh}$ from $6 \mathrm{~V}$ output of BUCK circuit. The system consumed $2209.92 \mathrm{mWh}$ from the rechargeable batteries. The hardware design of sink nodes as proposed in their paper used microcontroller (LPC2148), ZigBee (CC2530), GPRS (SIM300C), and Ethernet (ENC28J60) modules. The experiment lasted two weeks at test sites in the temperature range of $20^{\circ} \mathrm{C}$ to $28^{\circ} \mathrm{C}$. The previous designs were either restricted on the number of sensors for specific purpose or consumed relatively higher energy and their systems were evaluated at the ambient temperatures of more than $15^{\circ} \mathrm{C}$ (Gutierrez et al., 2014; Yan, Sun, \& Qian, 2013; Bengherbia et al., 2016; Fisher, \& Kebede, 2010; Yang, \& Tian, 2013).

The novelty of this research work lies in the design of S-Node by which the energy consumption has been brought down to $42.72 \mathrm{mWh}$ and field performance of MaxBotix Snow Depth Sensor has been improved by incorporating Euclidean Minimum Distance (EMD) Filter. Design methodology helps to monitor the snow environment with less energy consumption. Energy efficient sink node has been evaluated experimentally for long term at remote site in Indian Himalayas at low temperature up to $-9.8^{\circ} \mathrm{C}$.

The remaining article is framed as below.

- Section 2 describes the design approach and various hardware components used along with different modules/ components of S-node.

- Section 3 presents the results and discussion in detail regarding the present work.

- Section 4 summarizes the major findings as conclusion.

\section{Design Approach}

The energy efficient design of the proposed S-Node is explained in the following sub-sections of Major Hardware Components \& Design Methodology, Working Sequence and Modules/ Components.

\subsection{Major Hardware Components \& Design Methodology}

The S-Node is designed around ATmega2560 microcontroller of M/s Atmel Corporation which is used in Arduino Mega Shield and has 256kByte Flash Memory, 86 x I/O lines and 4 x USART ports. Due to better hardware and memory resources, S-Node can accommodate many COTS sensors at very small energy expense of $42.72 \mathrm{mWh}$ and has been successfully utilised in monitoring of snow environment at remote observatory by integration of Multi-parameter Sensor of M/s FRT, Model No. FWS500 and low-cost Snow Depth Sensor of M/s 
MaxBotix, Model No. MB7589 SCXL-WRT (hereafter, named as MaxBotix Sensor). Standard prototype kits of microcontroller are not optimized for the energy consumption and they consume much more power even if microcontroller is operated in sleep mode. Bengherbia et al. (2016) used FPGA board with power supply of $5000 \mathrm{mWh}$ and Yang, \& Tian (2013) used prototype kit of LPC2148 which operated at 368.32mAh. S-Node achieves lower energy consumption of $42.72 \mathrm{mWh}$ in standalone mode by employing following design methodology:

- Periodic snapping of the power lines of all modules except the RTC \& microcontroller with help of customized Main Power Module

- Selecting low power consuming hardware components

- Forcing the microcontroller in to power down mode when idle

- Using low duty cycle

- Using customized frame structure with positional encoding

\subsection{Working Sequence}

On triggering of hourly Alarm, RTC interrupts microcontroller by pulling down INT0 pin and ISR is executed. The warm-up time of $15 \mathrm{~s}$ is enabled to settle the signal of sensors to steady state output. The values of sensors are sampled and stored in SD card with date and time stamp. The data are transmitted through GSM link to central station at interval of one hour. It takes maximum 02 minutes for warm up, acquiring data, storing sample values and sending transmission through GSM module. At end of next hour RTC wakes up microcontroller. So, low duty cycle of $3.33 \%$ is achieved in reference to periodic transmission of one hour.

\subsection{Modules/ Components}

The S-Node relays the data of remote sensors nodes to the central station. For economical and quick turn out design time of S-Node, COTS Arduino shields are used. ATmega2560 microcontroller is the heart of S-Node which is used in Arduino Mega board, therefore, programmable by ArduinoIDE. A large number of $\mathrm{C} / \mathrm{C}++$ libraries are available at GitHub repositories for all the sub-modules of S-Node, thereby, reducing the effort in embedded programming. The current consumption of ATmega2560 in power down mode is small 7.0 $\mu \mathrm{A} @ 5 \mathrm{~V}$, $8 \mathrm{MHz}$ at operating temperature of $25^{\circ} \mathrm{C}$ and it works at lower temperatures upto $-40^{\circ} \mathrm{C}$. Though, ATmega328 has smaller current consumption $4.2 \mu \mathrm{A} @ 5 \mathrm{~V}, 8 \mathrm{MHz}$ in power down mode, however, ATmega2560 at clock frequency of $8 \mathrm{MHz}$ has been preferred for design of S-Node due to higher number of resources. The block diagram of the S-Node is given in Figure 1 and hardware layout of the prototype is shown in Figure 2.

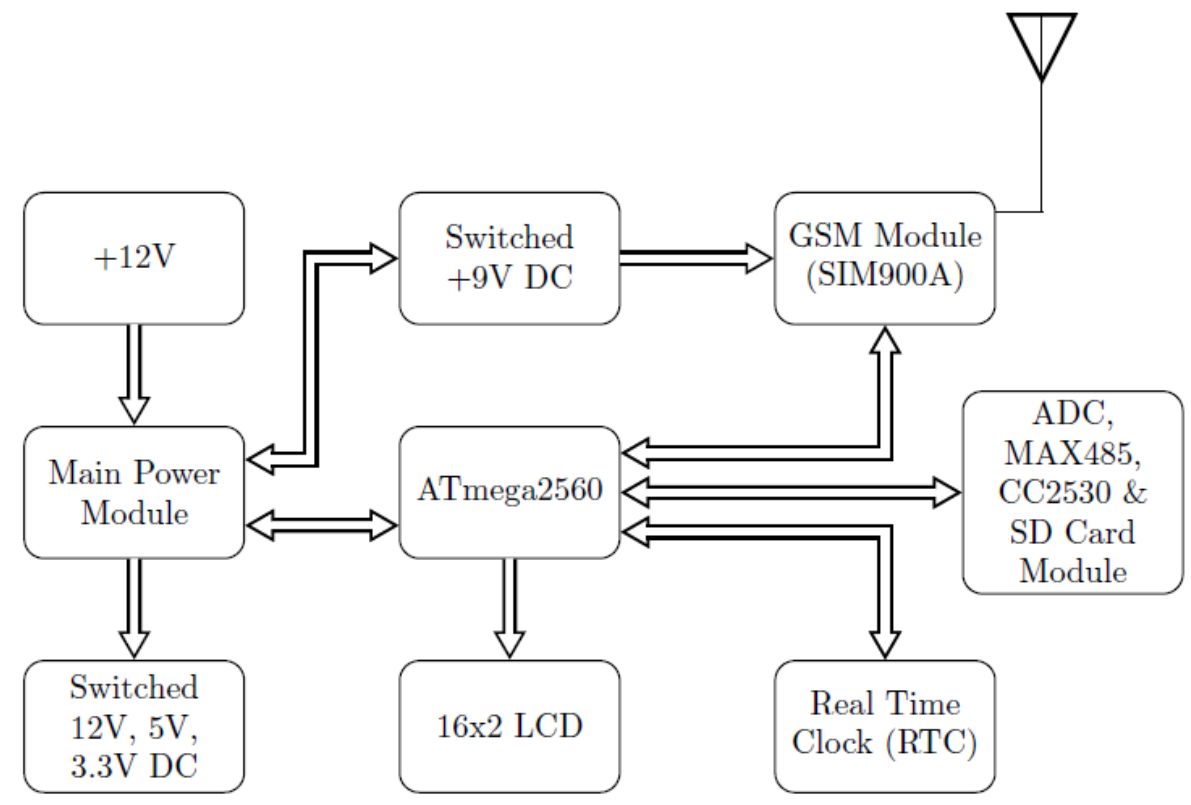

Figure 1. Block diagram of S-Node 


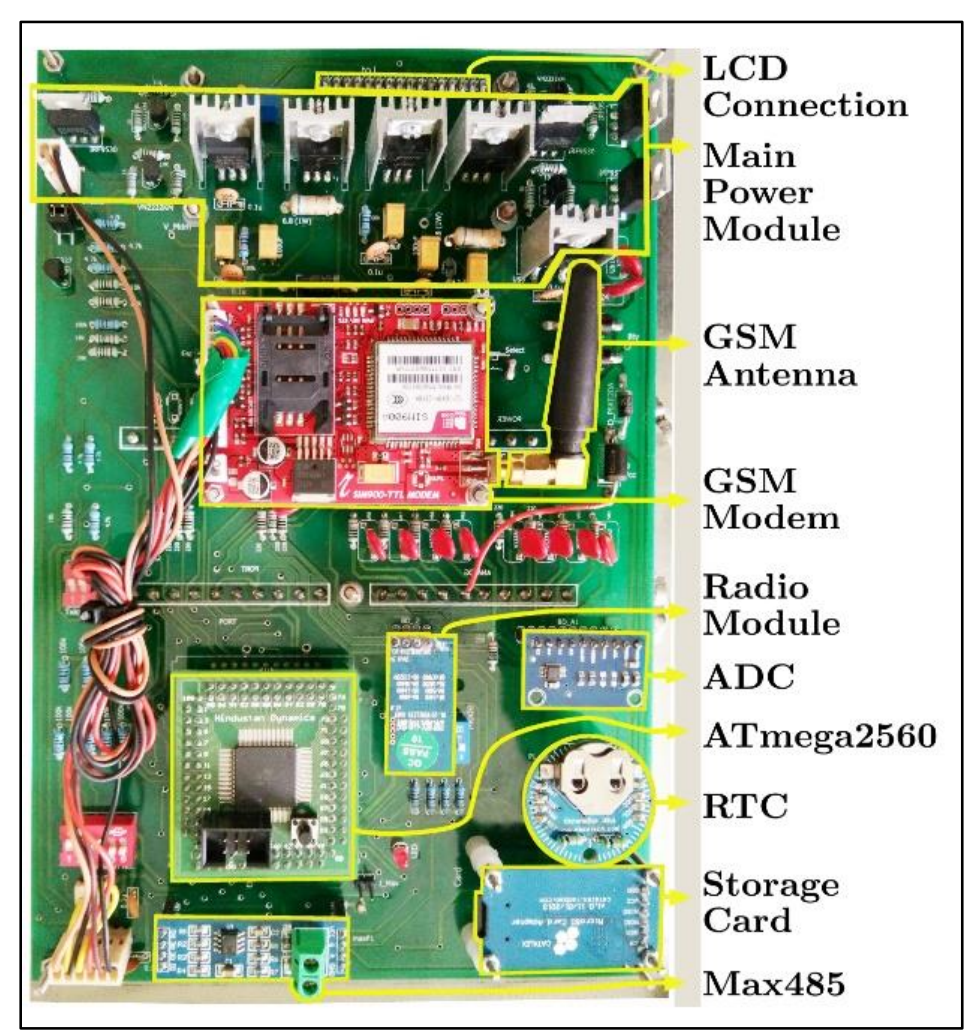

Figure 2. Hardware layout of S-Node

The details of customized module and standard modules are given in following sub-sections:

\subsubsection{Main Power Module}

Main Power Module is specially customized to implement the provision of switched powers to all modules and sensors. ATmega2560 controls the Main Power Module for activating/ snapping the power lines of the shields and sensors/ peripherals. Whole switching is achieved with combination of N-Channel Enhancement Mode MOSFET (VN2222LL) and P-Channel HEXFET Power MOSFET (IRF9530). Digital high signal at Gate Terminal of VN2222LL drives IRF9530 to pass the voltage at source terminal to the active load. Heat losses at higher currents are reduced as the source-drain resistance $(0.2 \Omega)$ of IRF9530 is very small. Linear regulators and IRF9530 are chosen in TO-220 package which helps in easy mounting of heat sinks. The Main Power Module derives switched voltages from $12 \mathrm{~V}$ external supply for operation of all the peripherals and sub-modules. LowDropout Voltage Regulator MIC2937A provides $+5 \mathrm{~V}$ round the clock to the microcontroller and RTC. The regulator has very low quiescent current of $160 \mu \mathrm{A}$, which ensures low power consumption of the S-Node. Fixed linear regulators LM1117I-3.3, LM7805CT and LM7809CT generate switched voltages of $+3.3 \mathrm{~V},+5 \mathrm{~V}$ and $+9 \mathrm{~V}$ to power Zigbee Module (CC2530), MaxBotix Sensor and GSM Module, respectively. Switched voltages of $+5 \mathrm{~V}$ and $+9 \mathrm{~V}$ are designed with external pass elements TIP125 to enhance the surge current capacity of the power system. Additionally, external voltage $(+12 \mathrm{~V})$ is bypassed to power up Multi-parameter Sensor (FWS500).

\subsubsection{Communication Module}

Communication segment has two sub-modules GSM SIM900A Shield and CC2530 Shield. GSM Module is built on low-cost dual band GSM/GPRS engine- SIM900A that works on frequencies 900/ 1800MHz. The module is directly operated with TTL voltage levels compatible to ATmega2560. The module typically consumes average current of $\approx 80.0 \mathrm{~mA}$ from $9 \mathrm{~V}$ and has intermittent high surge power needs for communicating with GSM network. AT-commands have been implemented on ATmega2560 for automatic network identification, registration and sending of SMS in ASCII mode. The module is connected to Serial1 port of ATmega2560. S-Node stores and sends the data to central station at one-hour interval as per frame structure given in Table 1. By using positional encoding and ignoring special characters, frame size has been brought down from 88 to 72 ASCII characters. As single SMS contains maximum 160 ASCII characters, so, the data of at least two hours are transmitted, thereby, providing redundancy of one hour in data transmission. Even if SMS is not received for one hour, the previous data can be replenished. The position of each field ASCII character helps to decode the data at central station. 
The node to sink node communication is with Zigbee compliant CC2530 radio module of Texas Instruments. The module is compliant to IEEE 802.15.4 standard. Serial2 port of ATmega2560 drives the Tx and Rx lines of CC2530 through the logic level translator which has been realized using 2N7000 N-Channel Enhancement Mode MOSFET. The power on switch of CC2530 Shield configures the radio module for Point-to-Point or Broadcasting Mode of transmission.

Table 1. Frame structure of storage $\&$ transmission

\begin{tabular}{llll}
\hline Description & Data Format & SSCII Size $^{*}$ & Remarks \\
\hline Start of Frame & 0 to Z & $1(1)$ & - \\
\hline Date Time & $\begin{array}{l}\text { Current Date Time } \\
\text { (YYYY-MM-DD HH:MN) }\end{array}$ & $15(12)$ & - \\
\hline Station No. & 0001 to 9999 & $4(4)$ & - \\
\hline Battery Voltage & 10.0 to 16.0 & $4(3)$ & - \\
\hline Ch1-Ch4 & 0.000 to 5.000 & $20(16)$ & Analog Channels \\
\hline Max1 - Max8 & 0.000 to 9.999 & $40(32)$ & MAX485 Channels \\
\hline End of Frame & FADE & $4(4)$ & - \\
\hline Total & & $\mathbf{8 8}(\mathbf{7 2})$ & \\
\hline
\end{tabular}

\section{* Positional Encoded Size in Brackets}

\subsection{Sensors \& Standard Modules}

Multi-parameter Sensor FWS500 has been selected viz-a-viz individual sensors for saving the energy and making the system compact. FWS500 connects on digital port MAX485 which provides Atmospheric Pressure (AP), Wind Speed (WS), Relative Humidity (RH), Wind Direction (WD) and Ambient Temperature (AT). Additionally, MaxBotix Sensor connects to S-Node to provide live standing snow readings. Ryan, Doeskin, \& Fassnacht (2008) had carried out the comparative evaluation of state-of-the-art ultrasonic snow depth sensors by installing the sensors of M/s Campbell Scientific and M/s Judd Communications at nine sites across the United States. These sensors are field proven, but they are expensive and consume much more energy. MaxBotix Sensor, currently deployed with S-Node, needs only $340 \mathrm{~mW}$ of power whereas; the snow depth sensors of M/s Judd Communications and M/s Campbell Scientific Instruments consume 600mW and 2250mW, respectively. Therefore, MaxBotix Sensor is suitable for energy constrained locations and also economical to deploy at mass scale. However, the performance of MaxBotix Sensor needs improvement with Euclidian Minimum Distance (EMD) Filter as highlighted in Section 3.3. The general purpose I/O lines have been used for integrating and configuring standard modules viz. DS3232SN-Real Time Clock, 32GB-SD Card, HD44780-16x2 LCD Display, 16 Bit ADC - ADS1115 and MAX485.

\subsection{Site Selection \& Sensor Parameters}

The design so realized had been subjected to long term testing at remote field site from 9th February to 8th May, 2017 at Dhundhi (Figure 3), which is located in Pir Panjal Mountain Range at Latitude (32 $\left.21^{\prime} 19.5^{\prime \prime} \mathrm{N}\right)$, Longitude $\left(77^{\circ} 07^{\prime} 42^{\prime \prime} \mathrm{E}\right)$ and Altitude of $3050 \mathrm{~m}$ above mean sea level. Compact visualization of all environmental parameters has been presented in Figure 4 in normalized scale for the period of evaluation i.e. from 09th Feb, 2017 to 08th May, 2017. S-Node processed raw value of MaxBotix Sensor to derive filtered value using EMD filter and transmitted both values to central station.

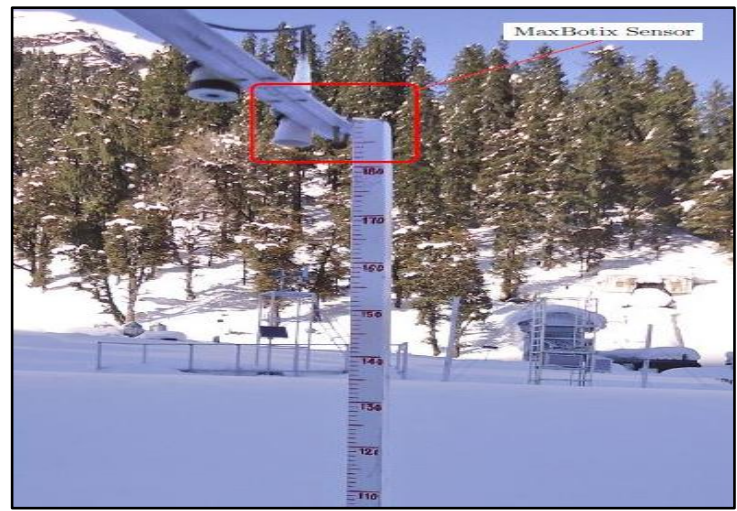

Figure 3. MaxBotix sensor at field site 


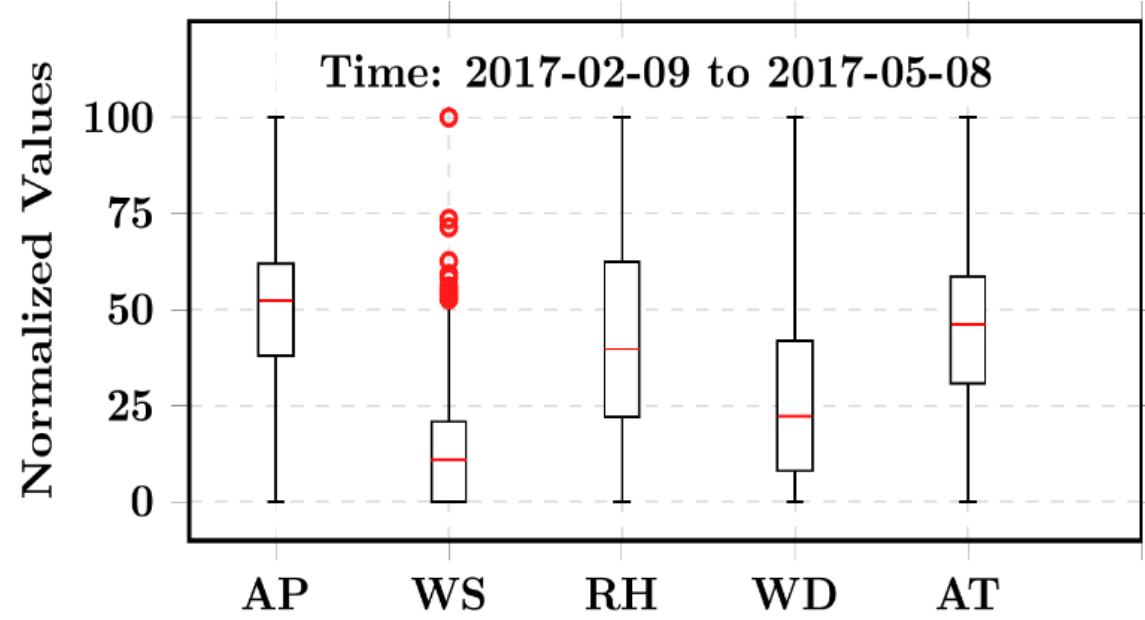

Figure 4. Recorded data of multi-sensor

\section{Results and Discussion}

\subsection{Data Recording \& Analysis of MaxBotix Sensor}

MaxBotix sensor was vertically mounted at height of $3.50 \mathrm{~m}$ above the ground surface at Dhundhi field site. The snowpack had already built up to $1.74 \mathrm{~m}$ in the previous snow-spells. Channel No.-1 of 16-Bit ADC (ADS1115) Shield measured the output of sensor. GSM Shield sent hourly data to the central station in ASCII encoded format of Table 1. The decoded data are plotted in the Figure 5 which shows the variation of output voltage of MaxBotix Snow Depth Sensor from 9th February to 8th May, 2017. After 9th February, 2017, the SNode tracked all five snow spells successfully without single failure and online data were recorded at distant central station $\approx 320 \mathrm{~km}$ away from field site. The snow depth declined when snowpack settled under the gravitational force or melted due to rise in temperature and increased whenever snow-fall occurred. Reflecting surface of snow appeared nearer to the sensor if snow depth was increasing.

Thereby, standing snow depth is inversely proportional to the voltage output of the sensor. By subtracting the MaxBotix output voltage from mounting height of sensor i.e. 3.5, the snow depth is calculated. Frequent transitions from low to high and vice versa are observed in the output voltage of MaxBotix sensor during the snow-fall. Sensor also showed four spurious events $\{$ S1, S2, S3, S4 $\}$ during the evaluation period from 9th February, 2017 to 8th May, 2017. The performance of the sensor is improved by deploying Euclidean Minimum Distance Filter.

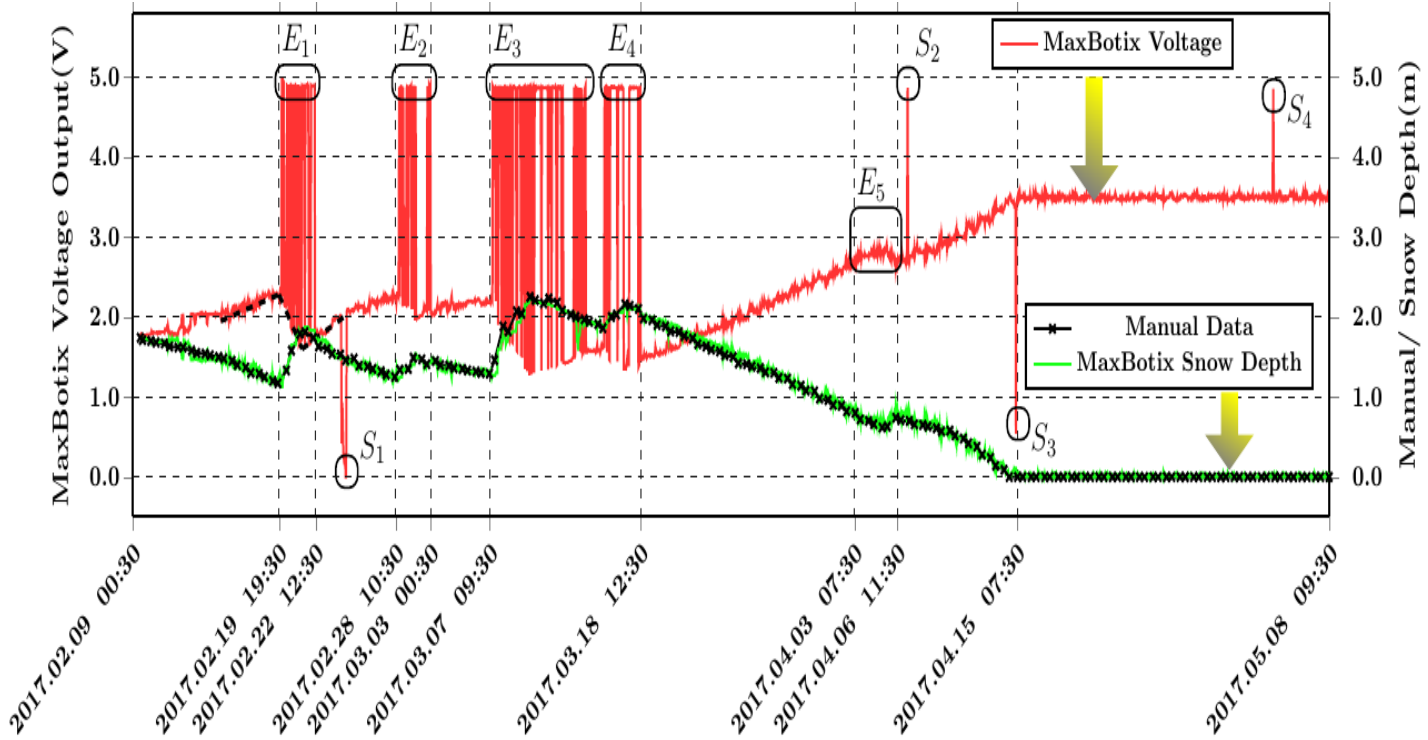

Date Time

Figure 5. Long term recorded and EMD filtered data at field site 
Table 2. Hourly energy consumption of proposed modules of S-Node

\begin{tabular}{|c|c|c|c|c|}
\hline Module & Time (Minutes) & $\begin{array}{l}\text { Current } \\
(m A @ V)\end{array}$ & Consumption & $\begin{array}{l}\text { Average. Energy } \\
\text { Consumption }(\mathrm{mWh})\end{array}$ \\
\hline LDO-MIC2937A & 60 & $0.160 @ 12 \mathrm{~V}$ & & 1.92 \\
\hline RTC-DS3232SN & 60 & $0.325 @ 5 \mathrm{~V}$ & & 1.63 \\
\hline ADC-ADS1115 & 02 & $0.180 @ 5 \mathrm{~V}$ & & 0.03 \\
\hline $\begin{array}{l}\text { ATmega2560 } \\
\text { (Power Down) }\end{array}$ & 58 & $0.007 @ 5 \mathrm{~V}$ & & 0.03 \\
\hline $\begin{array}{l}\text { ATmega2560 } \\
\text { (Active) }\end{array}$ & 02 & $24.00 @ 5 \mathrm{~V}$ & & 4.00 \\
\hline LM1117I-3.3 & 02 & $5.00 @ 12 \mathrm{~V}$ & & 2.00 \\
\hline LM7805CT & 02 & $5.00 @ 12 \mathrm{~V}$ & & 2.00 \\
\hline LM7809CT & 02 & $5.00 @ 12 \mathrm{~V}$ & & 2.00 \\
\hline GSM Module & 02 & 80.00@9V & & 24.00 \\
\hline MAX485 Module & 02 & $0.50 @ 5 \mathrm{~V}$ & & 0.08 \\
\hline CC2530 Module & 02 & $39.60 @ 3.3 \mathrm{~V}$ & & 4.36 \\
\hline SD Card Module & 02 & $4.00 @ 5 \mathrm{~V}$ & & 0.67 \\
\hline Sub Total & & & & 42.72 \\
\hline FWS500 & 02 & $20.00 @ 12 \mathrm{~V}$ & & 8.00 \\
\hline MaxBotix Sensor & 02 & $68.00 @ 5 \mathrm{~V}$ & & 11.33 \\
\hline Total & & & & 62.05 \\
\hline
\end{tabular}

\subsection{Energy Consumption in S-Node}

By employing above said design methodology, working sequence and modules/ shields, the overall energy consumption of S-Node has been reduced. The S-Node achieves energy consumption of $42.72 \mathrm{mWh}$ in standalone mode which is much lesser than 80mAh of Gutierrez et al. (2014) and 368.32mAh of Yang, \& Tian (2013). Similarly, S-Node consumes $62.05 \mathrm{mWh}$ when MaxBotix Sensor and Multi-parameter Sensor are connected for monitoring of snow environment by employing hourly transmission of data to the central station. The calculation details of energy consumption are summarized in Table 2.

\subsection{Euclidean Minimum Distance (EMD) Filter}

The frequent transitions in output signal $(>4.5 \mathrm{~V})$ of the MaxBotix Sensor are expected due to the reflections of ultrasonic signals from the falling snow-flakes. The probable reason of inconsistency is attributable to the most likely filter implemented in the firmware of sensor which prefers the signal of closest target when it receives multiple reflections of similar amplitude. In order to improve the error so associated, the readings are staggered throughout the hour by taking 5 samples at an interval of 5 minutes. At end of hour, sample size [S] of 60 values is available for processing. As snowfall is non-uniform, there are some instants in $[\mathrm{S}]$ when reflecting surface distance is measured accurately. Sensor value having minimum Euclidean distance to previous value is selected as final value and higher values are discarded as spurious values. Methodology of recording is given below:-

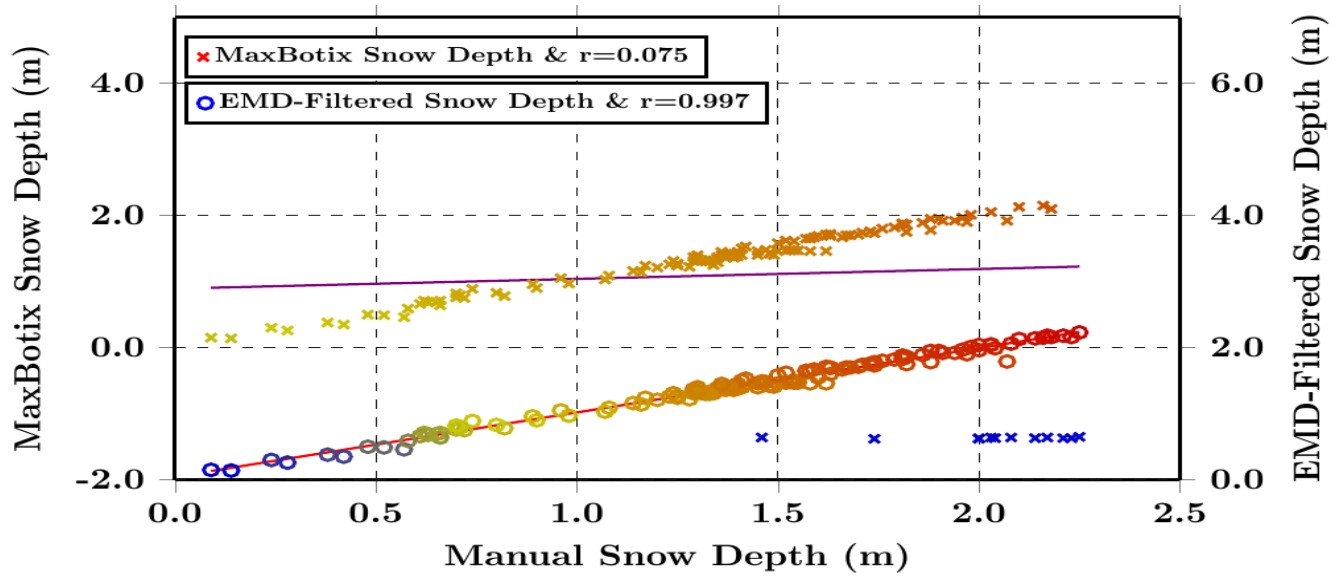

Figure 6. Scatter diagram of raw and filtered data with manual data 
Let $X_{\text {old }}$ be the initial value before snowfall starts and $X_{\text {new }}$ be the subsequent hourly reading to be transmitted. On hourly trigger, take sample size of 5 readings [S] for measurement consistency. If the median value in sample $[\mathrm{S}]$ is not high $(<4.5 \mathrm{~V})$ then finalize the median as new sample to be transmitted, otherwise, increase sample rate to 5 minutes till end of hour and select the value having minimum Euclidean distance to previous value of sensor. The variation of filtered snow depth after incorporation of above process sequence is also shown in Figure 5. The algorithm for implementation of the process sequence in embedded program of SNode, is given in Algorithm 1.

Algorithm 1. Filter algorithm for MaxBotix snow depth sensor

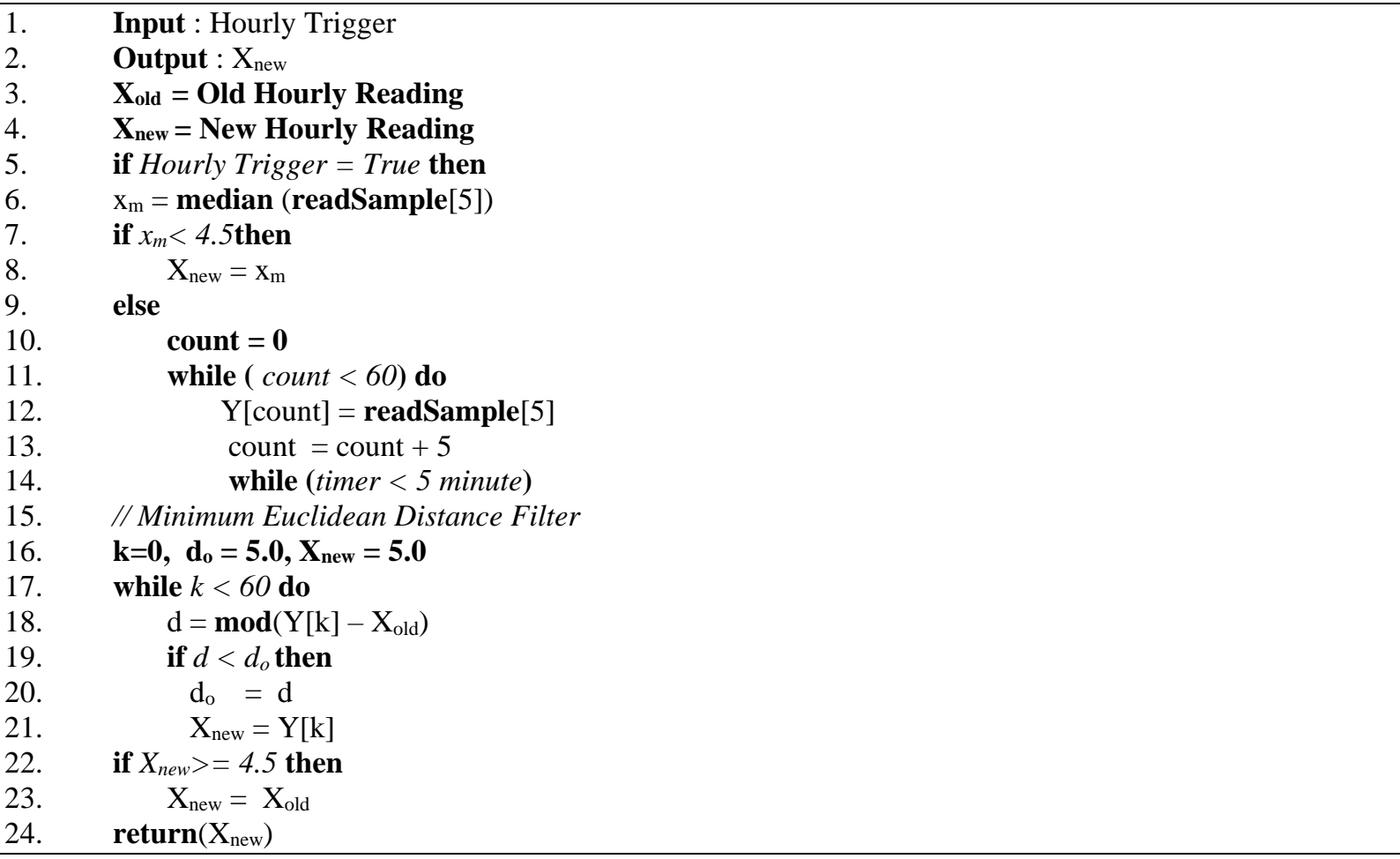

\subsection{Comparison of MaxBotix Data}

The MaxBotix Sensor was mounted at height of 3.50m in Dhundhi field site. So, output readings of MaxBotix Sensor were offset adjusted and compared with manually recorded readings at 3-GMT and 12-GMT. Figure 5 plots the variation in the snow depth of MaxBotix Sensor and manual snow depth. Scatter diagrams have been plotted for the better insight of measurements. Figure 6 shows the variation of raw snow depth of MaxBotix sensor w.r.t. manual snow depth. Due to spurious values of raw data, low correlation coefficient (0.075) is observed. Figure 6 also shows the variation of filtered data w.r.t. manual snow depth after incorporation of EMD filter. We observe high correlation of $0.997(\mathrm{RMSE}=0.054)$ due to the removal of spurious values.

Design of S-Node is able to achieve low power consumption of $42.72 \mathrm{mWh}$ (Figure 7) which is significantly lower than the previous such designs reported by Gutierrez et al., 2014; Bengherbia et al., 2016 and Yang, \& Tian, 2013. For ensuring ruggedity at low temperature, the designed configuration of S-Node and sensors have been evaluated for long time in actual field location. Dhundhi site of evaluation witnessed minimum temperature of $-9.8^{\circ} \mathrm{C}$ in the evaluation period. Unexpected variations in the values of MaxBotix Snow Depth Sensor have been observed in the field location. So, Minimum Euclidean distance filter was incorporated in S-Node which improved the performance of MaxBotix Snow Depth Sensor. Raw data of MaxBotix Sensor w.r.t. actual snow depth showed weak linear relationship with significant outliers and offset values, thereby showing less value (Figure 6) of correlation coefficient (0.075). However, the minimum Euclidean distance filtered data showed strong, positive and linear relationship with high value (Figure 6) of correlation coefficient (0.997) when compared with the actual snow depth. Therefore, by incorporating the proposed design methodology and combination of sensors, the snow environment can be monitored with less expense of energy. 


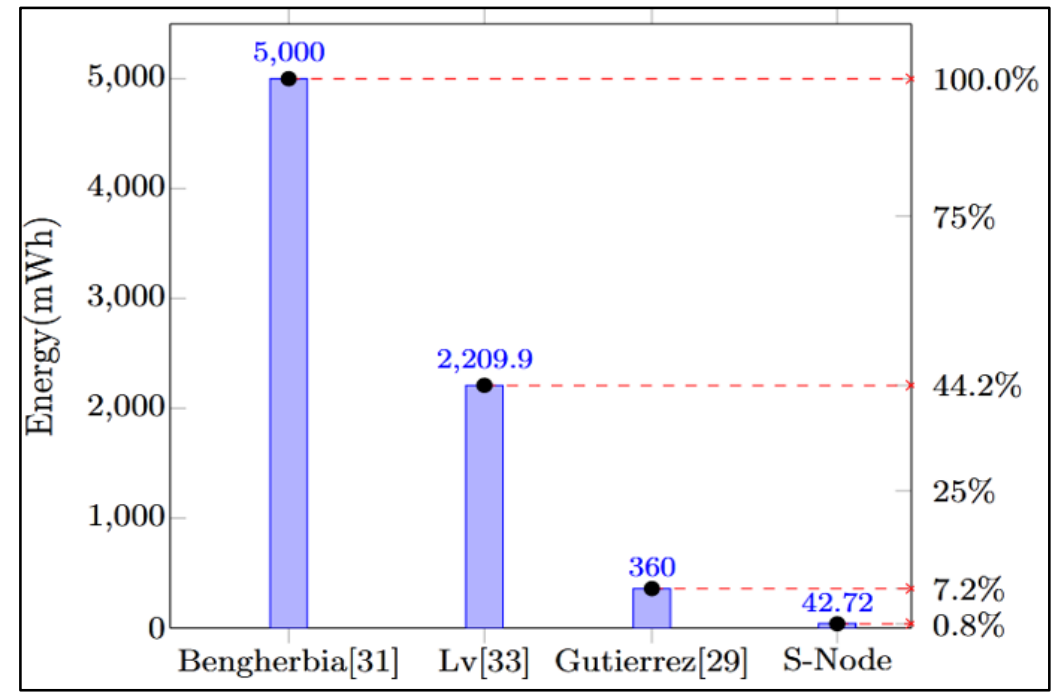

Figure 7. Comparison of energy consumption

\section{Conclusions}

This paper reported the development of S-Node which is designed around COTS shields and open-source Arduino-IDE. S-Node is able to integrate many types of environmental sensors. It can supply switched power to all types of sensors as per the operating voltages with the customized Power Module. S-Node is able to achieve the power consumption of $42.72 \mathrm{mWh}$ which is significantly lower than the previous work. It consumes $62.05 \mathrm{mWh}$ energy to operate for one hour while recording environmental parameters from Multi-parameter Sensor (FWS500) and MaxBotix Snow Depth Sensor. S-Node worked reliably at remote observatory at low temperature of $-9.8^{\circ} \mathrm{C}$. All events of snowfall and day-to-day variations were recorded in the period of evaluation. By using customized frame structure with positional encoding and energy efficient design, consistent data were recorded at remote and central stations. The performance of low cost MaxBotix sensor is further improved by incorporating Euclidean Minimum Distance filter which improves the correlation to 0.997 between the output of MaxBotix Sensor and actual data. Finally, the proposed hardware configuration of sink node (SNode) and sensors can be deployed for practical and economical monitoring of snow environment at energy constrained remote locations like Pir Panjal Mountain Range of Indian Himalayas.

\section{References}

1. Akyildiz, I. F., Su, W., Sankarasubramaniam, Y., \& Cayirci, E. (2002). A survey on sensor networks. IEEE Communications Magazine, 40(8), 102-114.

2. Al-Karaki, J. N., Ul-Mustafa, R., \& Kamal, A. E. (2004). Data aggregation in wireless sensor networks - exact and approximate algorithms. Workshop on High Performance Switching and Routing, (Phoenix AZ, USA) 2004, 241-245.

3. Allippi, C., Anastasi, G., Francesco, M. D., \& Roveri, M. (2009). Energy management in wireless sensor networks with energy-hungry sensors. IEEE Transactions on Instrumentation and Measurement, 12(2) 16-23.

4. Bai, K., \& Tepedelenlioglu, C. (2008). Distributed detection in UWB wireless sensor networks. IEEE International Conference on Acoustics, Speech and Signal Processing (Las Vegas NV, USA) 2008, 2261-2264.

5. Bengherbia, B., Chadli, S., Zmirli, M. O., \& Toubal, A. (2016). A MicroBlaze based WSN sink node using XBee transceiver. 8th International Conference on Modelling, Identification and Control (Algiers, Algeria) 2016, 831-834.

6. Chen, M., Kwon, T., Mao, S., Yuan, Y., \& Leung, V. (2008). Reliable and energy efficient routing protocol in dense wireless sensor networks. Sensor Networks, 4(1) 104-117.

7. Corke, P., Wark, T., Jurdak, R., Hu, W., Valencia, P., \& Moore, D. (2010). Environmental wireless sensor networks. Proceeding of the IEEE, 98(11) 1903-1917.

8. Erlacher, F., Weber, B., Fischer, J. T., \& Dressler, F. (2016). AvaRange using sensor network ranging techniques to explore the dynamics of avalanches. 12th Annual Conference on Wireless On-demand Network Systems and Services (Cortina d'Ampezzo, Italy) 2016, 120-123.

9. Fisher, D. K., \& Kebede, H. (2010). A low-cost microcontroller-based system to monitor crop temperature and water status. Computers and Electronics in Agriculture, 74(1) 168 - 173. 
10. FRT-FWS500, http://www.fronttech.com.cn (Accessed on 3rd Nov, 2020).

11. Gutiérrez, J., Villa-Medina, J. F., Nieto-Garibay, A., \& Porta-Gándara, M. Á. (2014). Automated irrigation system using a wireless sensor network and GPRS module. IEEE Transactions on Instrumentation and Measurement, 63(1) 166-176.

12. Heinzelman, W. B., Chandrakasan. A. P., \& Balakrishnan, H. (2002). An application-specific protocol architecture for wireless microsensor networks. IEEE Transactions on Wireless Communications, 1(4) 660-670.

13. Heinzelman, W. R., Chandrakasan, A., \& Balakrishnan, H. (2000). Energy-efficient communication protocol for wireless microsensor networks. Proceedings 33rd Annual Hawaii International Conference on System Sciences (Maui HI, USA) 2000, 2, 1-10.

14. Jiang, X., Polastre, J., \& Culler, D. (2005). Perpetual environmentally powered sensor networks. Fourth International Symposium on Information Processing in Sensor Networks, (Boise ID, USA) 2005, 463-468.

15. Kandris, D., Tsioumas, P., \& Tzes, A., Nikolakopoulos, G., \& Vergados, D. D. (2009). Power conservation through energy efficient routing in wireless sensor networks. Sensors, 9(9) 7320 7342.

16. Khalil, M. I., Hossain, M. A., Haque, M. J., \& Hasan, M. N. (2017). EERCMAC: energy efficient receiver centric MAC protocol for wireless sensor network. IEEE International Conference on Imaging, Vision \& Pattern Recognition (University of Dhaka, Bangladesh) 2017, 1-5.

17. Kolios, P., Panayiotou, C., Ellinas, G., \& Polycarpou, M. (2016). Data-driven event triggering for IoT applications. IEEE Internet of Things Journal, 3(6) 1146-1158.

18. Kompis, C., \& Sureka, P. (2010). Power management technologies to enable remote and wireless sensing. Technical Report (ESP KTN Teddington, UK) 2010.

19. Manjeshwar, A., \& Agrawal, D. P. (2001). TEEN: A routing protocol for enhanced efficiency in wireless sensor networks. Proceedings 15th International Parallel and Distributed Processing Symposium (San Francisco CA, USA) 2001, 2009-2015.

20. Manjeshwar, A., \& Agrawal, D. P. (2002). APTEEN: a hybrid protocol for efficient routing and comprehensive information retrieval in wireless. Proceedings 16th International Parallel and Distributed Processing Symposium (Ft. Lauderdale FL, USA) 2002.

21. MaxBotix, MB7589 SCXL-WRT Ultrasonic Sensor, https://www.maxbotix.com (Accessed on 3rd Nov, 2020).

22. Mirza, D., Owrang, M., \& Schurgers, C. (2005). Energy-efficient wakeup scheduling for maximizing lifetime of IEEE 802.15.4 networks. Proceedings 1st International Conference on Wireless Internet (Budapest, Hungary) 2005, 130-137.

23. Oliveira, L. M., \& Rodrigues, J. J. (2011). Wireless sensor networks: a survey on environmental monitoring. Journal of Communications, 6(2) 143-151.

24. Pantazis, N. A., Nikolidakis, S. A., \& Vergados, D. D. (2013). Energy-efficient routing protocols in wireless sensor networks: a survey. IEEE Communications Surveys \& Tutorials, 15(2) 551-591.

25. Park, C., \& Chou, P. H. (2006). AmbiMax: autonomous energy harvesting platform for multisupply wireless sensor nodes. 3rd Annual IEEE Communications Society on Sensor and Ad Hoc Communications and Networks (Reston VA, USA) 2006, 168-177.

26. Raghunathan, V., Ganeriwal, S., \& Srivastava, M. (2006). Emerging techniques for long lived wireless sensor networks. IEEE Communications Magazine, 44(4) 108-114.

27. Ryan, W. A., Doesken, N. J., \& Fassnacht, S. (2008). Evaluation of ultrasonic snow depth sensors for U.S. snow measurements. Journal of Atmospheric and Oceanic Technology, 25, 667-684.

28. Sardini, E., \& Serpelloni, M. (2011). Self-powered wireless sensor for air temperature and velocity measurements with energy harvesting capability. IEEE Transactions on Instrumentation and Measurement, 60(5) 1838-1844.

29. Sharma, V., Mukherji, U., \& Joseph, V. (2010). Optimal energy management policies for energy harvesting sensor nodes. IEEE Transactions on Wireless Communications, 9(4) 1326-1336.

30. Simjee, F. I., \& Chou, P. H. (2008). Efficient charging of super-capacitors for extended lifetime of wireless sensor nodes. IEEE Transactions on Power Electronics, 23(3) 1526-1536.

31. Sinha, A., \& Chandrakasan, A. (2001). Dynamic power management in wireless sensor networks. IEEE Design Test of Computers, 18(2) 62-74.

32. Subudhi, B., \& Pradhan, R. (2013). A comparative study on maximum power point tracking techniques for photovoltaic power systems. IEEE Transactions on Sustainable Energy, 4(1) 8998.

33. Yan, R., Sun, H., \& Qian, Y. (2013). Energy-aware sensor node design with its application in wireless sensor networks. IEEE Transactions on Instrumentation and Measurement, 62(5) 11831191. 
34. Yang, Lv., \& Tian, Yu. (2013). Design and application of sink node for wireless sensor network. The International Journal for Computation and Mathematics in Electrical and Electronic Engineering, 32(2) 531-544.

35. Yang, Y., \& Cardei, M. (2010). Delay-constrained energy efficient routing in heterogeneous wireless sensor networks. Sensor Networks, 7(4) 236-247. 